\title{
Emerging data on the treatment of multiple sclerosis
}

\author{
Andrei Ciuhan', Lacramioara Perju-Dumbrava², Nicoleta Tohanean² \\ ${ }^{1}$ Faculty of Medicine, "Iuliu Hatieganu" University of Medicine and Pharmacy, Cluj-Napoca, Romania \\ ${ }^{2}$ Neuroscience Department, "Iuliu Hatieganu" University of Medicine and Pharmacy, Cluj-Napoca, Romania
}

\begin{abstract}
Background and objectives. In the past few years, a myriad of new studies were aimed to find better ways to manage MS. As a result, a bunch of new molecules were found to have good efficacy, therefore FDA and EMA approved a series of treatments in the last few years, the last one receiving green light from EMA on March 30th, 2021 (Ofatumumab Kesimpta ${ }^{\circledR}$ ). The aim of this study was to evaluate and classify three of the newest drugs approved by the FDA and EMA. Material and methods. All the studies were chosen on the basis of pre-determined inclusion criteria and in accordance with PRISMA guidelines. We searched Pubmed and Cochrane Library for all studies published up until the end of 2020. For the data analysis we used Metalnsight ${ }^{\circledR}$, a statistical web-based tool for meta-analyses and NMAs performing both Frequentist and Bayesian hierarchical model analyses, each one being seen as a sensitivity check for the other.

Outcomes. The best therapeutic agent in reported efficacy amongst the three analyzed was Ofatumumab, ranked first in hierarchy, Ozanimod and Cladribine following in the second and third place, respectively.

Conclusions. According to ABN's 2015 guidelines, Cladribine was ranked between the most effective medicines for the treatment of MS; given the results from this study, other two may be considered as high efficacy alongside Natalizumab, Alemtuzumab and Ocrelizumab.
\end{abstract}

Keywords: multiple sclerosis, network meta-analysis, immunomodulatory drugs,

Frequentist NMA, Bayesian NMA

\author{
Abbreviations \\ NMA - Network Meta-Analysis; \\ MS - multiple sclerosis; \\ ABN - Association of British Neurologists; \\ CNS - central nervous system; \\ EMA - European Medicines Agency; $\quad$ ARR - annualized relapse ratio; \\ FDA - Food and Drug Administration; DMT - disease-modifying treatments
}

\section{INTRODUCTION}

Multiple sclerosis is a chronic neurodegenerative disorder of unknown etiology. There are now over 150 years since Charcot's neurological triad was first described, albeit given the complexity of the disease, the attempts of finding the missing link which, alongside genetic proneness and environmental factors could be responsible for the autoimmune assault against CNS, reached no definite answer (1).

A recent epidemiological study comprising prevalence data from 75 countries found a 50\% higher prevalence reported in 2020 than in 2013 (2), a wor- ryingly insight. Likewise, a systemic analysis of all epidemiological data from 1990 until 2016 showed consistency regarding anterior findings in the latitude gradient distribution of SM, higher mortality in women, higher prevalence in whites and lower in children (3).

The last five years were prosperous. Four new drugs showed good efficacy in randomized clinical trials; therefore, FDA and EMA approved their use. Cladribine, Ofatumumab, Ozanimod and Ocrelizumab (including a shorter two-hour infusion dosed twice yearly), despite their proved efficacy against placebo or an active first-generation drug, lack evidence in head-to-head trials. 
The rationale of this study was to generate a hierarchy based on indirect data comparison of the newly approved drugs (Cladribine, Ofatumumab, Ozanimod) using the ARR reported in the primary studies and following an analytical technique known as an extension of a conventional meta-analysis to a network meta-analysis.

\section{MATERIAL AND METHODS}

The data from randomized controlled trials conducted before January $1^{\text {st }}, 2021$, underwent statistical analysis in a NMA for ARR. We looked for all studies which directly compared Cladribine, Ofatumumab and Ozanimod with an active or inactive agent and had, as a primary or secondary outcome, efficacy evaluation through ARR.

\section{Sources}

We used Pubmed and Cochrane Library for literature search purposes and via the latter we found additional information of finished and ongoing studies from EMBASE, Clinicaltrials.gov and ICTRP (International Clinical Trials Platform).

\section{Search strategy}

We obtained the search results using the PICOT search strategy in MEDLINE's search engine and Cochrane Library. Searches with synonymous terms were performed automatically.

\section{Study selection}

Inclusion and exclusion criteria were necessary for study selection. We excluded the studies which had no ARR calculated as an outcome, non-randomized-controlled trials, follow-up under six months, different drug administration protocol and other studies not relevant for the current analysis. All search results were carefully evaluated and selected in preparation for data extraction.

\section{Data extraction and manipulation}

All necessary data required to perform this analysis was extracted and modified in compliance with international protocols and recommendations for systematic reviews and NMAs.

For each therapeutic arm we extracted aARR (adjusted ARR) expressed by M (Mean), SD (Standard Deviation) and $\mathrm{N}$ (Number of patients). None of the studies reported the SD value, therefore we extracted it indirectly using Cochrane's Software - Review Manager (4) based on the 95\%CI (Confidence Interval) associated with $\mathrm{M}$ and the demonstrated symmetry around the mean. Likewise, where needed, we deducted SD using SE (standard error) or ARR's MD (mean difference) and the associated $p$ value.

\section{Quality assessment}

All studies were evaluated in order to eliminate the risk of bias. Thereinafter, we used the Cochrane Collaboration's tool for assessing risk of bias in RCTs and the criteria for judging risk of bias for each domain (5).

\section{Data analysis}

We analyzed our data using MetaInsight tool - a web-based platform for conducting NMAs (6). The strategy was to perform a two-way check of the results using R packages netmeta and gemtc, specifically both Frequentist and Bayesian hierarchical model analyses. The two statistical models differ, albeit the results should be similar, thus confirming the results' validity.

\section{OUTCOMES}

We ran a search applying the described strategy in the specified databases and found 393 studies, out of which 9 were included in this analysis. The PRISMA flowchart in figure 1 recounts reasons for the exclusion of some studies.

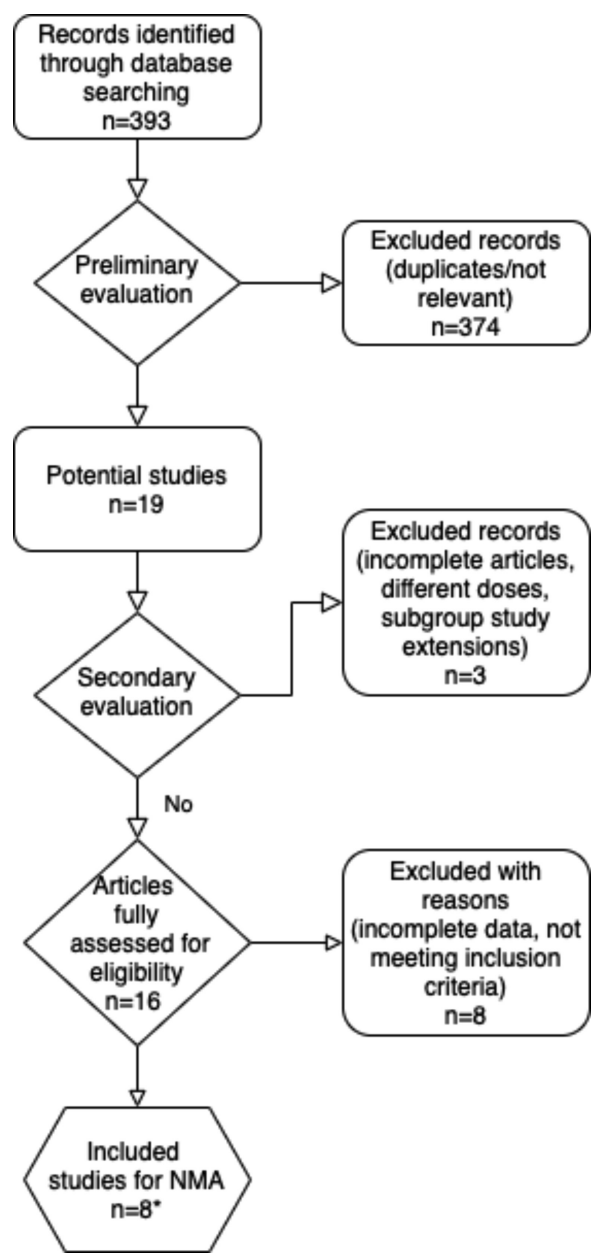

FIGURE 1. PRISMA flowchart. One study consists of two interventions ASCLEPIOS I and ASCLEPIOS II which have been treated as individual studies, therefore resulting 9 total studies in the network 
TABLE 1. Selected studies 1

\begin{tabular}{|c|c|c|c|c|c|}
\hline & Author & Year & Study & $\begin{array}{c}\text { Treatment/ } \\
\text { Dose }\end{array}$ & References \\
\hline 1 & Cohen JA & 2019 & RADIANCE & Ozanimod 1 mg vs. Interferon b1a IM 30 ug & 7 \\
\hline 2 & Comi G & 2019 & SUNBEAM & Ozanimod 1 mg vs. Interferon b1a IM 30 ug & 8 \\
\hline 3 & Vollmer TL & 2014 & BRAVO & Interferon b1a IM 30 ug vs. Placebo & 9 \\
\hline 4 & Jacobs LD & 1996 & MSCRG & Interferon b1a IM 30 ug vs. Placebo & 10 \\
\hline 5 & Giovannoni G & 2010 & CLARITY & Cladribina 3.5 mg/kg vs. Placebo & 11 \\
\hline 6 & Hauser SL & 2020 & ASCLEPIOS I & Ofatumumab 20 mg vs. Teriflunomid 14 mg & 12 \\
\hline 7 & Hauser SL & 2020 & ASCLEPIOS II & Ofatumumab 20 mg vs. Teriflunomid 14 mg & 12 \\
\hline 8 & O'Conner P & 2011 & TEMSO & Teriflunomid 14 mg vs. Placebo & 13 \\
\hline 9 & Confavreux C & 2014 & TOWER & Teriflunomid 14 mg vs. Placebo & 14 \\
\hline
\end{tabular}

The selected studies can be found in table 1 .

The preliminary results showed the network being connected, thus permitting us to proceed with the analysis. The total number of possible pairwise comparisons was 15 , out of which 5 with direct data, and comprised a total of 7031 patients in the network. In figure 2 we obtained an overview for all studies included in the NMA based on the input of the extracted data.

The forest plot allowed us to observe the evidence available for each comparison and to examine the heterogeneity between studies.

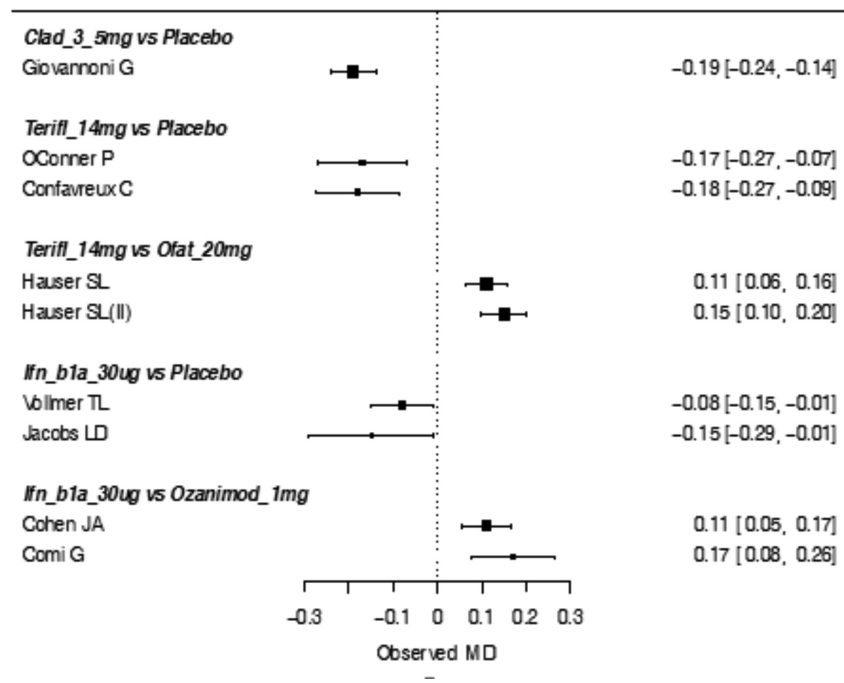

FIGURE 2. Individual study results (for all studies) grouped by treatment comparison

The network plot (Figure 3) provided a visual display of the head-to-head compared interventions in the trials. There have been 6 interventions, 9 pairwise comparisons and one sub-network.

\section{Frequentist NMA results}

In the associated forest plot (Figure 4) we displayed the pooled effect estimates and 95\% confidence intervals for all interventions compared to the reference treatment, in this case - placebo.

The Frequentist method allowed us to evaluate all point estimates resulting from pairwise me-

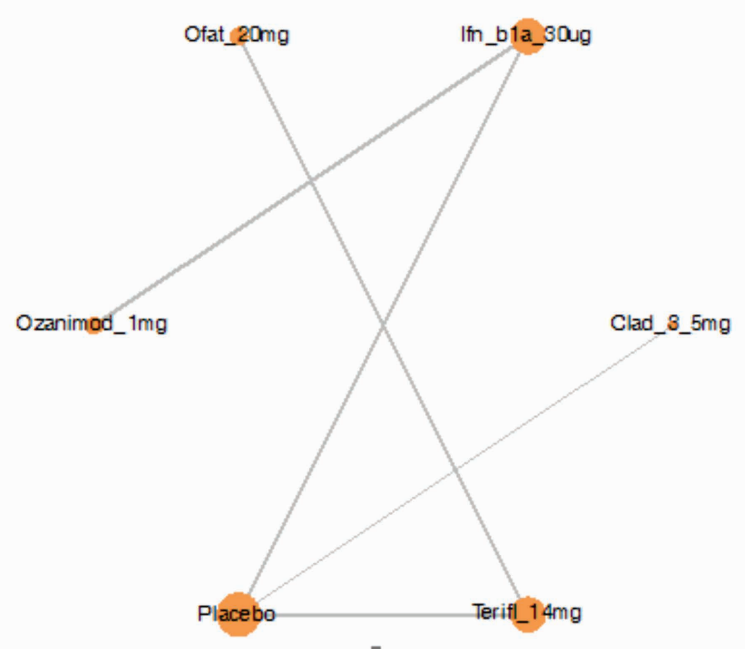

FIGURE 3. Network plot for all studies

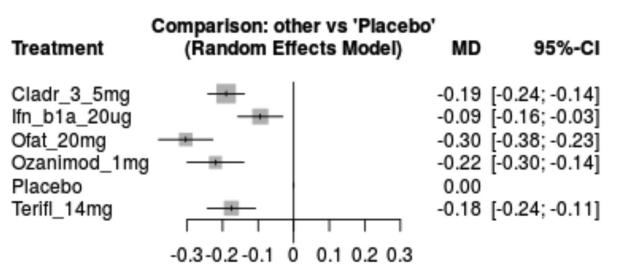

FIGURE 4. Frequentist forest plot. Between-study SD: 0; no. of studies: 9; no. of drugs: 6; all outcomes are versus the reference treatment, Placebo

ta-analyses - only from direct head-to-head evidence - as well as treatment comparisons obtained from NMA, with indirect data. All values represent the pooled mean difference (MD) and the associated CI.

Inconsistency was assessed for all studies and did not appear to be any important estimated differences between direct and indirect evidence.

\section{Bayesian hierarchical model results}

Similar to the Frequentist result, the Bayesian result (figure 5) also shown Ofatumumab (Ofat_20mg) as having the best pooled estimate 
compared with placebo but with larger uncertainty depicted by the $95 \%$ credible interval.

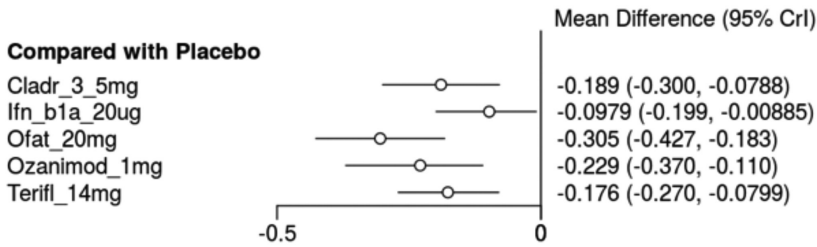

FIGURE 5. All studies: Bayesian random effect consistency model forest plot results. Between-study SD: $0.4 ; 95 \% \mathrm{Cl}$ $[0,0.12]$

The convergence assessment plots for Bayesian NMA model showed that the simulated models converged. Per-arm residual deviance for all studies showed contribution around one, indicating good fit in the model and lower residuals. Also, the analysis did not indicate evidence of inconsistency for any study arm.

\section{DISCUSSION}

The network consisted of nine studies which have been integrated in an extended statistical analysis. The two analytical methods reached similar outcomes, displaying the superiority of all therapeutic agents over placebo and ranking them according to ARR's reduction (figure 6).

The Bayesian inconsistency indexes showed similar influence of the studies on the overall NMA results, although the algorithms had limited power in assessing inconsistency in the Frequentist analytical method.

\begin{tabular}{|cc|}
\hline Rank & Intervention \\
\hline 1 & Ofat_20mg \\
\hline 2 & Ozanimod_1mg \\
\hline 3 & Cladr_3_5mg \\
\hline 5 & Terifl_14mg \\
\hline 6 & Ifn_b1a_20ug \\
\hline
\end{tabular}

FIGURE 6. Ranking with all studies - NMA median rank chart
The ABN guidelines for prescribing DMTs in SM (15), published in 2015, categorized the approved immunomodulators for SM in high and medium efficacy. Ofatumumab, Ozanimod and Cladribine could be considered as high efficacy alongside Natalizumab and Alemtuzumab, whereas Teriflunomide and Interferon beta 1a could be considered as medium efficacy.

The new approved DMTs have not yet been included in a revised guideline by the ABN or other association, wherefore there is a need for updated guidelines which could have significant impact on patient's disease activity and compliance on the premises of a more facile administration regimen and the reported high efficacy.

Considering the lack of evidence concerning the new approved DMTs, new studies should aim to compare directly in head-to-head trials the efficacy of the drugs in order to fill the informational gap existing in the current medical knowledge.

The breadth of this study was limited to the comparison of three therapeutic agents, and we generated a hierarchy based solely on the efficacy reported in the primary studies through ARR, without considering other efficacy or tolerability indexes and excluding other possible variables in the making of therapeutic strategies.

\section{CONCLUSIONS}

From the available data analyzed, we found Ofatumumab in doses of 20 milligrams administered in a monthly subcutaneous dose as being the most efficacious for the treatment of multiple sclerosis. The following ranked treatments were Ozanimod (1 milligram), Cladribine (3.5 milligrams), Teriflunomide (14 milligrams) and Interferon beta 1a (30 micrograms). The sensitivity analysis performed showed the results are robust.

The results look very promising and, with the extended range of treatments made available, the patients might experience less relapses and an overall good quality of life over the years lived with the disease. 


\section{REFERENCES}

1. Libner CD, Salapa HE, Levin MC. The Potential Contribution of Dysfunctional RNA-Binding Proteins to the Pathogenesis of Neurodegeneration in Multiple Sclerosis and Relevant Models. Int J Mol Sci. 2020 Jun 27;21(13):E4571.

2. Walton C, King R, Rechtman L, Kaye W, Leray E, Marrie RA, et al. Rising prevalence of multiple sclerosis worldwide: Insights from the Atlas of MS, third edition. Mult Scler. 2020 Dec;26(14):1816-21.

3. Wallin MT, Culpepper WJ, Nichols E, Bhutta ZA, Gebrehiwot TT, Hay SI, et al. Global, regional, and national burden of multiple sclerosis 1990-2016: a systematic analysis for the Global Burden of Disease Study 2016. Lancet Neurol. 2019 03;18(3):269-85.

4. Review Manager (RevMan) [Computer program]. Version 5.4, The Cochrane Collaboration, 2020.

5. Higgins JPT, Thomas J, Chandler J, Cumpston M, Li T, Page MJ, et al. Cochrane Handbook for Systematic Reviews of Interventions. 2nd Edition. Chichester (UK): John Wiley \& Sons, 2019.

6. Owen RK, Bradbury N, Xin Y, Cooper N, Sutton A. Metalnsight: An interactive web-based tool for analyzing, interrogating, and visualizing network meta-analyses using R-shiny and netmeta. Res Syn Meth. 2019;10:569-581.

7. Cohen JA, Comi G, Selmaj KW, Bar-Or A, Arnold DL, Steinman L, et al. Safety and efficacy of ozanimod versus interferon beta-1a in relapsing multiple sclerosis (RADIANCE): a multicentre, randomised, 24-month, phase 3 trial. Lancet Neurol. 2019 11;18(11):1021-33.

8. Comi G, Kappos L, Selmaj KW, Bar-Or A, Arnold DL, Steinman L, et al. Safety and efficacy of ozanimod versus interferon beta-1a in relapsing multiple sclerosis (SUNBEAM): a multicentre, randomised, minimum 12-month, phase 3 trial. Lancet Neurol. 2019 11;18(11):1009-20.

9. Vollmer TL, Sorensen PS, Selmaj K, Zipp F, Havrdova E, Cohen JA, et al. A randomized placebo-controlled phase III trial of oral laquinimod for multiple sclerosis. J Neurol. 2014 Apr;261(4):773-83.

10. Jacobs LD, Cookfair DL, Rudick RA, Herndon RM, Richert JR, Salazar $A M$, et al. Intramuscular interferon beta-1a for disease progression in relapsing multiple sclerosis. The Multiple Sclerosis Collaborative Research Group (MSCRG. Ann Neurol. 1996 Mar;39(3):285-94.

11. Giovannoni G, Comi G, Cook S, Rammohan K, Rieckmann P, Soelberg Sørensen $P$, et al. A placebo-controlled trial of oral cladribine for relapsing multiple sclerosis. N Engl J Med. 2010 Feb 4;362(5):416-26.

12. Hauser SL, Bar-Or A, Cohen JA, Comi G, Correale J, Coyle PK, et al. Ofatumumab versus Teriflunomide in Multiple Sclerosis. N Engl J Med. 2020;383(6):546-57.

13. O'Connor P, Wolinsky JS, Confavreux C, Comi G, Kappos L, Olsson TP, et al. Randomized trial of oral teriflunomide for relapsing multiple sclerosis. N Engl J Med. 2011 Oct 6;365(14):1293-303.

14. Confavreux C, O'Connor P, Comi G, Freedman MS, Miller AE, Olsson $T P$, et al. Oral teriflunomide for patients with relapsing multiple sclerosis (TOWER): a randomised, double-blind, placebo-controlled, phase 3 trial. Lancet Neurol. 2014 Mar;13(3):247-56.

15. Scolding N, Barnes D, Cader S, Chataway J, Chaudhuri A, Coles A, et al. Association of British Neurologists: revised (2015) guidelines for prescribing disease-modifying treatments in multiple sclerosis. Pract Neurol. 2015 Aug;15(4):273-9. 